Ebisu Ebisu

Études japonaises Études japonaises

$53 \mid 2016$

1914-1918, une guerre mondiale ? La perspective japonaise

\title{
Catherine MAYAUX (textes réunis et présentés par), La fleur cachée du nô
}

Paris, Honoré Champion, 2015, 164 p.

\section{Magali Bugne}

\section{(2) OpenEdition}

\section{Journals}

Édition électronique

URL : https://journals.openedition.org/ebisu/1944

DOI : 10.4000/ebisu.1944

ISSN : 2189-1893

Éditeur

Institut français de recherche sur le Japon à la Maison franco-japonaise (UMIFRE 19 MEAE-CNRS)

Édition imprimée

Date de publication : 10 décembre 2016

Pagination : 291-295

ISSN : $1340-3656$

\section{Référence électronique}

Magali Bugne, «Catherine mayaux (textes réunis et présentés par), La fleur cachée du nô », Ebisu [En ligne], 53 | 2016, mis en ligne le 10 décembre 2016, consulté le 08 novembre 2021. URL : http:// journals.openedition.org/ebisu/1944; DOI : https://doi.org/10.4000/ebisu.1944 
fois avant de parvenir à la délivrance, gageons que nombreux seront les lecteurs qui auront besoin de plusieurs lectures pour parvenir à un entendement correct du texte de Myōe. En échange des efforts demandés, l'ouvrage de Frédéric Girard offre un éclairage sur la notion de foi - une question, comme le dit l'auteur au début de l'ouvrage, à la fois centrale, complexe et multiforme dans le bouddhisme - et, tout aussi précieux parce que rare en langue occidentale, une fenêtre sur un bout du monde intellectuel de l'époque de Kamakura.

\section{Didier Davin \\ Kokubungaku kenkyū shiryō-kan \\ 国文学研究資料館 \\ (Institut national de littérature japonaise)}

1. Dans "Chūsei ni okeru kenmitsu taisei no tenkai »中世における顕密体制の 展開 (Développement du système kenmitsu au Moyen Âge), Nihon chüsei no kokka to shūkyō 日本中世の国家と宗教 (L'État et la religion du Japon médiéval), Tokyo, Iwanami shoten 岩波書店, 1975.

2. Sueki Fumihiko 末小文美士, Kamakura bukkyō keisei ron - shisōshi no tachiba kara 鎌倉仏教形成論一思想史の立場から (L'établissement du bouddhisme de Kamakura: le point de vue de l'histoire de la pensée), Kyoto, Hōzōkan 法藏館, 1998.

3. Le lien entre les enjeux idéologiques $\mathrm{du} \mathrm{Xx}^{\mathrm{e}}$ siècle et les études bouddhiques est souligné dans de nombreuses études. Signalons ici simplement, comme exemple récent, l'ouvrage d'Orion Klautau, Kindai Nihon shisō to shite no bukkyōshigaku 近代
日本思想としての仏教史学 (L'historiographie du bouddhisme comme pensée japonaise moderne), Kyoto, Hōzōkan 法藏館, 2012.

4. Un moine de la secte Kegon à l'époque de Kamakura (1185-1333), Myōe (1173-1232) et le Journal de ses rêves, Paris, Publications de l'École française d'Extrême-Orient, vol. CLX, 1990. Signalons la parution récente d'une édition richement commentée de ce texte: Okuda Isao 奥田勲, Hirano Tae 平野多恵, Maekawa Ken.ichi 前川健一, Myōe shōnin yume no ki-yakuchū 明恵上人 夢記一訳注 (Le journal des rêves de l'éminent Myōe commenté et annoté), Tokyo, Bensei shuppan 勉誠出版, 2015.

(9) Catherine Mayaux

(textes réunis et présentés par), La fleur cachée du nô, Paris, Honoré Champion, 2015, 164 pages.

Les textes réunis et présentés ici par Catherine Mayaux relèvent le défi de proposer une synthèse allant de l'origine historique et esthétique du nô au Japon jusqu'à ses différentes réceptions dans le théâtre occidental. L'ouvrage peut se diviser en deux grandes parties: une première consacrée à l'émergence et la réception moderne du théâtre nô dans la culture japonaise et une seconde tournée vers l'Occident dans laquelle est proposée une analyse de 
la réception et des adaptations de cette forme théâtrale au cours du $\mathrm{Xx}^{\mathrm{e}}$ siècle.

Le recueil s'ouvre sur un article de Nishino Haruo ("Le poète dramatique Zeami, ses œuvres et ses théories artistiques "), ancien directeur du Centre de recherche sur le nô de l'université Hōsei, qui présente une analyse détaillée de l'œuvre du dramaturge Zeami (1363-1443), chef de la troupe Kanze et fondateur du «nô d'apparition» (mugen nō 夢幻能). Ce type de nô se caractérise par une double structure séparant la pièce en deux actes. Le deuxième acte, qui se déroule entièrement dans le rêve du deutéragoniste (waki), permet la révélation de la nature surnaturelle du personnage principal (shite). Cet être extraordinaire, esprit ou fantôme souffrant de malemort, revit alors dans le monde onirique ses derniers instants. Par ce déroulement temporel régressif, Zeami s'éloigne de la temporalité réaliste qui avait façonné le théâtre dialogique du début du $\mathrm{XIV}^{\mathrm{e}}$ siècle pour accéder à un art à forte connotation symbolique.

De la courtisane oubliée du nô Hanjo au fantôme du nô Izutsu dont même la mort ne parvient pas à faire disparaître l'attachement amoureux, Nishino met en avant la douce nostalgie sentimentale des fantômes de femmes créés par Zeami. Chacune de ces femmes incarne un idéal esthétique dont le "charme subtil " (yügen 幽玄) vise à faire s'épanouir dans le cœur du spectateur une émotion profonde, désignée dans les traités du maître sous la mystérieuse appellation de "fleur" (hana 花). C'est ce concept que Kuroda Akinobu ("Le geste dans le théâtre nô ") analyse en détail au travers d'une réflexion portant sur le geste dans le théâtre nô à partir d'un postulat phénoménologique sur la mise en scène. En premier lieu, il isole deux types de "fleur» dans les écrits de Zeami : la "fleur authentique» (makoto no hana 真の花) et la « fleur du moment "(jibun no hana 時分の花). Selon Zeami, la «fleur authentique " représente pour l'acteur le résultat d'une profonde maîtrise des arcanes de son art alors que la «fleur du moment" est due au charme passager de la jeunesse et est donc vouée à disparaître avec le temps. Kuroda propose une relecture de ces deux notions au premier abord antinomiques en les interprétant comme concurrentes et complémentaires. Il isole ainsi dans les derniers traités de Zeami une troisième "fleur" cachée qu'il choisit de nommer «la fleur du présent éternel ». Située au-delà de toute temporalité et de toute forme figée, cette «fleur» tient de ce qui échappe à toute identification substantielle car elle est en vérité "l'insolite en toute chose » (p. 33). Sans substance, elle se transmet de cœur à cour et permet à 
l'acteur de talent de se réinventer perpétuellement sur scène pour continuer à émerveiller le spectateur.

Mais la capacité d'un acteur à renouveler son jeu ne saurait expliquer à elle seule la persistance du nô dans la culture japonaise. Aussi, dans son article, Jean-Michel Butel ("Vieillir ensemble sans confusion: l'idéal amoureux chanté par la pièce Takasago ") s'intéresse-t-il à un des exemples les plus marquants de l'assimilation du nô à la société japonaise moderne: la récitation de vers tirés de la pièce Takasago lors des cérémonies de mariage. Pour Butel, la mécanique du couple mise en scène dans Takasago entre en résonance avec la conception idéalisée du couple qui accompagna l'élaboration de la cérémonie de mariage moderne au Japon dans la deuxième moitié du $\mathrm{xx}^{\mathrm{e}}$ siècle ${ }^{1}$.

Comme l'expliquait Nishino dans le premier article de cet ouvrage, les nô mettant en scène des femmes prennent traditionnellement pour thème les affres de la passion. L'attachement amoureux de la femme à son époux la tourmente et l'empêche de trouver la paix. Or Takasago, nô votif à fonction propitiatoire, fait ici figure d'exception dans le répertoire du nô en substituant à la passion amoureuse un modèle de couple apaisé "dont chaque pôle reste distinct» (p. 53).
Butel analyse cette distinction au travers d'une étude textuelle détaillée de la pièce qui met en évidence le mouvement unilatéral des échanges du couple sur scène. Allant toujours de l'homme vers la femme, l'élément masculin prévaut sur l'élément féminin qui se contente de remplir le rôle passif mais complémentaire d'« accompagnant» (tsure).

La deuxième partie du recueil est tournée vers l'Occident et présente des articles traitant de la courte mais dense histoire de la réception du nô d'apparition de Zeami en dehors des frontières du Japon. Nishino Ayako (" L'histoire de la réception du nô en Occident et son adaptation par Yeats, Pound, Claudel et Brecht ") met en évidence la réutilisation de la mécanique du rêve de Zeami dans les œuvres de grands écrivains occidentaux. Ezra Pound (1885-1972) s'inspire du nô Nishikigi de Zeami pour composer Tristan, réutilisant ainsi le monde onirique pour mettre en scène les moments les plus intenses de la vie des protagonistes. Chez William Butler Yeats (1865-1939), la double structure du nô se politise avec la composition en 1917 de The dreaming of the bones (Ce que rêvent les os) juste après le soulèvement de Pâques contre les Anglais. Il donne ainsi naissance à ce que la critique yeatsienne nomme le dreaming back, technique qui consiste à faire 
apparaître en rêve certaines scènes de la vie terrestre d'un mort pour lui permettre de faire pénitence. Si cette mécanique est également reprise par Paul Claudel (1868-1955), la pénitence des personnages est remplacée par « des héros chrétiens qui accèdent au Salut » (p. 68). Le rêve et son cortège de chimères se transforment sur scène en véhicules de la révélation divine dont l'apparition onirique de l'ange dans le Soulier de satin reste un des plus beaux exemples.

Louvrage présente également une copie du Nô de Paul Claudel, rédigé en 1926, et dont la phrase liminaire "le drame, c'est quelque chose qui arrive, le Nô, c'est quelqu'un qui arrive " (p. 75) définissait en vérité bien plus le nô d'apparition de Zeami que le théâtre nô dans son ensemble. Ce texte s'accompagne d'une analyse de Chūjō Shinobu ("Notes sur Nô de Paul Claudel ") qui part à la recherche de l'originalité du vocabulaire claudélien. Au travers d'un lexique savamment composé, il fait apparaître la vision toute singulière de Claudel sur ce théâtre auquel il superpose le "drame sacré " de la messe (p. 84). Loin de la critique savante, Claudel développe sa propre lecture esthético-spirituelle du nô dans lequel l'acteur officie comme un ministre du culte et les spectateurs, ses ouailles, procèdent à la communion pendant le spectacle ${ }^{2}$.
Pour Dominique Millet-Gérard ("Claudel et le nô : sources, méditations, intuitions "), qui se consacre à une analyse de la pantomime de la princesse dans Tête d'Or, cette lecture claudélienne du nô détaillée par Chūjō dépasse le domaine de l'écriture pour s'étendre jusque dans l'espace scénique. Au travers d'une étude des didascalies, il dévoile l'instauration d'un véritable monde imaginaire où la lenteur hiératique des gestes de la princesse fait plonger le spectateur dans un "drame somnambulique" se rapprochant de ce qu'il nomme le "pacte hypnotique" (p. 134) du nô. L'article de Philippe Forest ("Quelqu'un qui revient, notes sur le nô »), qui clôt le recueil, propose une réflexion plus générale sur le théâtre nô en soulignant l'importance de son caractère allusif, véritable source créatrice pour les auteurs, qui contribue à sa manière au développement de l'imaginaire.

Le présent ouvrage offre donc un large panorama allant du développement du théâtre de Zeami, exception régionale devenue théâtre national, à son adaptation dans le théâtre occidental au cours du $\mathrm{xx}^{\mathrm{e}}$ siècle. Si l'influence de l'œuvre de Zeami sur la dramaturgie occidentale est rendue dans toute sa mesure, il aurait été intéressant que soit évoquée son influence sur le monde du spectacle japonais qui est complètement 
passée sous silence. L'ouvrage propose cependant un travail d'ensemble rigoureux qui vient éclairer l'art du nô sous des angles multiples.

Magali Bugne Université de Strasbourg

1. Comme le rappelle à juste titre l'auteur, la bulle économique japonaise favorisa le développement d'une véritable industrie du mariage dont la ville éponyme de Takasago ne manqua pas de s'emparer en s'autoproclamant en 1988 « cité de la noce » (buraidaru toshi ブライダル都市). Il est cependant difficile de déterminer comment Takasago en est venu à être chanté lors d'un mariage, fruit d'un heureux hasard ou d'une association naturelle, ou peut-être même un mélange des deux.

2. Cette vision du théâtre nô suit les écrits de Noël Péri dont les articles et traductions de pièces, publiés de 1909 à 1921 dans le Bulletin de l'École française d'Extrême-Orient, furent compilés sous la forme d'un recueil en 1921 intitulé Cinq nô: drames lyriques japonais. Pour plus de détails sur cette vision du nô comme théâtre religieux et médiumnique, voir la préface de François Lachaud dans Le théâtre nô : études sur le drame lyrique japonais, Paris, École française d'ExtrêmeOrient, coll. réimpressions, 2004 : p. 5-11.
(D) Bernard Thomann, La naissance de l'État social japonais. Biopolitique, travail et citoyenneté dans le Japon impérial (1868-1945), Paris, Les presses de Sciences Po, 2015, 450 pages.

Bernard Thomann présente, dans son ouvrage, les politiques sociales qui se sont succédées de l'ère Meiji à la fin de la Seconde Guerre mondiale. Lopinion selon laquelle les fondements de l'État social japonais ont émergé après la Seconde Guerre mondiale est souvent reprise à l'étranger ${ }^{1}$ et au Japon même. La thèse de l'auteur est que l'ampleur et la profondeur des politiques et de leur mise en œuvre, ainsi que l'intensité des débats sur les questions sociales remettent en question cette opinion.

La première partie est consacrée aux politiques sanitaires et sociales qui accompagnèrent l'ouverture du Japon. Les mécanismes traditionnels de protection et d'assistance, basés sur la solidarité au sein des communautés villagoises, ne disparurent pas mais ils perdirent de leur centralité par rapport à des politiques plus centralisées. Leur présence continua à être d'autant plus nécessaire que les politiques publiques d'assistance aux 